\title{
Erratum: Connection between entanglement and the speed of quantum evolution [Phys. Rev. A 72, 032337 (2005)]
}

\author{
J. Batle, M. Casas, A. Plastino, and A. R. Plastino \\ (Received 16 February 2006; published 17 April 2006)
}

DOI: 10.1103/PhysRevA.73.049904 PACS number(s): 03.67.Mn, 89.70.+c, 03.65. - w, 02.50. $-\mathrm{r}, 99.10 . \mathrm{Cd}$

In Sec. II, Fig. 1 should be replaced by a new Fig. 1. This, in turn, originates small changes in the comments placed below Eq. (14). The specific origin of the errors in the previous (wrong) version of Fig. 1 was equation

$$
\Gamma=\sqrt{C^{2}} / 2
$$

appearing in the second line after Eq. (14). This equation, corresponding to the upper curve in the previous Fig. 1, is wrong because it was wrongly derived from Eq. (13) (which is indeed correct). A similar mistake led to Eq. (26) which must also be deleted. It affects the old Fig. 2.

According to the new Fig. 1, for all values of the concurrence $C \in[0,1]$ the largest possible evolution time is given by $\tau / T_{\min }=\sqrt{2}$. This maximum value corresponds to $\alpha=\pi$ and $\Gamma=1 / 4$ [see Eq. (14)]. On the other hand, the minimum possible value of $\tau / T_{\min }$ is a monotonically decreasing function of $C$. Consequently, the difference between the maximum and the minimum evolution times augments as the concurrence grows.

In Sec. III, Fig. 2 must be replaced by a new Fig. 2 as well, without changes in the caption. Furthermore, Eq. (23) must now be changed to

$$
T_{\min }=\frac{\pi \hbar}{4 \epsilon \sqrt{\Gamma}} .
$$

As in the previously mentioned case of two distinguishable particles (qubits), when we have two identical bosons the maximum evolution time is again (for all values of $C \in[0,1]$ ) given by $\tau / T_{\min }=\sqrt{2}$. As a consequence, Eq. (26) must now be deleted.

Summing up, the amendments that have to be made in the paper are as follows: Figs. 1 and 2 have to be replaced by the corresponding new ones; equation $\Gamma=\sqrt{C^{2}} / 2$ in the second line after Eq. (14) has to be deleted; Eq. (23) has to be modified; and Eq. (26) has to be deleted.

The main conclusions of the paper remain unchanged. Indeed, for systems of either two qubits or two identical bosons, the lower bound for the evolution time to an orthogonal state is a monotonically decreasing function of the concurrence. All separable states $(C=0)$ that evolve to an orthogonal state do so in the slowest ("worst") possible way, associated with $\tau / T_{\min }=\sqrt{2}$. In order to achieve faster ("better") evolution speeds (associated with smaller values of $\tau / T_{\min }$ ) one necessarily needs to increase the concurrence $C$ (that is, the amount of entanglement of the state). To achieve the fastest kind of possible evolution $\left(\tau / T_{\min }=1\right)$, states of maximum entanglement $(C=1)$ are required.

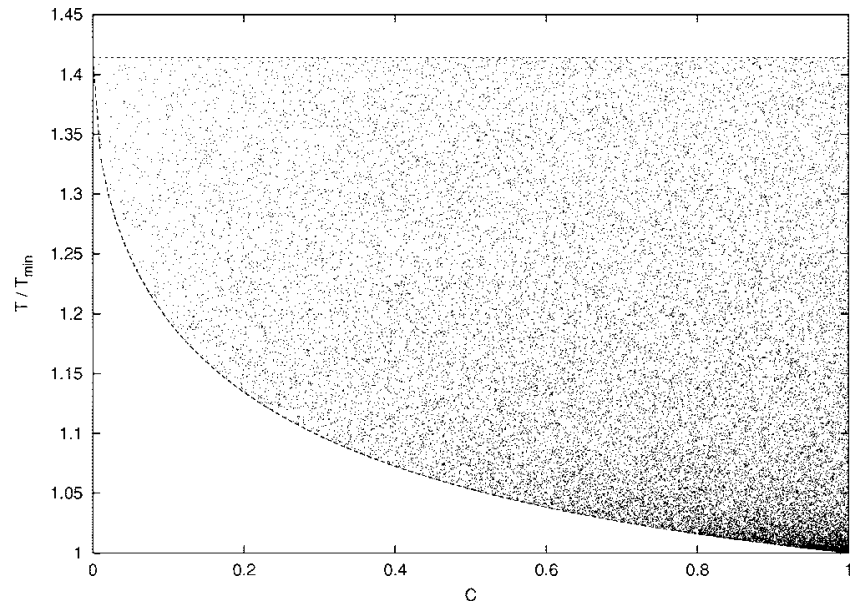

FIG. 1. Curves in the $\left(C, T / T_{\min }\right) \equiv\left(C, \tau / T_{\min }\right)$ plane corresponding, for each value of $C$, to the states of two (distinguishable) qubits with maximum and minimum $\tau / T_{\min }$. The points represent randomly generated individual states that evolve to an orthogonal state. All depicted quantities are dimensionless.

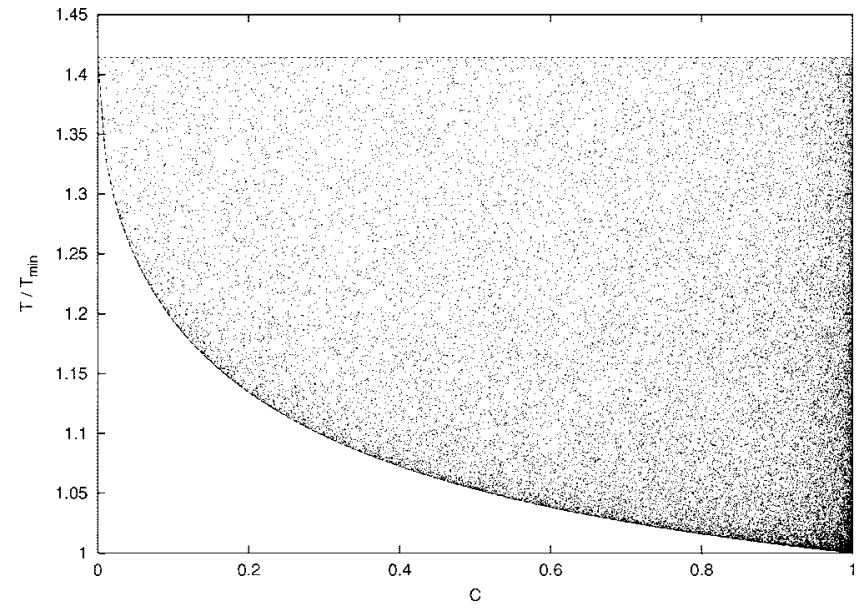

FIG. 2. Curves in the $\left(C, T / T_{\text {min }}\right) \equiv\left(C, \tau / T_{\text {min }}\right)$ plane corresponding, for each value of $C$, to the states of two bosons with maximum and minimum $\tau / T_{\min }$. The points represent randomly generated individual states that evolve to an orthogonal state. All depicted quantities are dimensionless. 